\title{
THE OCTOBER MEETING IN COLLEGE PARK
}

The five hundred seventeenth meeting of the American Mathematical Society was held at the University of Maryland, College Park, Maryland, on Saturday, October 22, 1955. The meeting was attended by about 175 persons, including 145 members of the Society.

By invitation of the Committee to Select Hour Speakers for Eastern Sectional Meetings Professor Paul Olum of Cornell University delivered an address entitled The fundamental problem of topology for 2-manifolds at a general session presided over by Professor L. W. Cohen. Sessions for contributed papers were held in the morning and afternoon, presided over by Professors I. N. Herstein, G. S. S. Ludford, and C. O. Oakley.

Abstracts of the papers presented follow. Those having the letter " $t$ " after their numbers were read by title. Where a paper has more than one author, that author whose name is followed by " $(\mathrm{p})$ " presented it.

\section{Algebra AND Theory of Numbers}

\section{W. E. Baxter: Lie simplicity of a special class of associative rings.}

In a forthcoming paper $\mathrm{I}$. N. Herstein proves that if $A$ is a simple ring of characteristic different from 2 and 3 , then the only proper Lie ideals of $[A, A]$ are contained in the center of $A$. In the present paper, the situations when $A$ has characteristic 2 and characteristic 3 are studied and Herstein's results are extended to these cases. In fact it is proved that the proper Lie ideals of $[A, A]$ are contained in the center except for the case $A=2 \times 2$ matrices over a field of characteristic 2 . These results are then applied to extend results of Hattori and Iwahori on invariant subrings of simple rings with descending chain conditions. (Received September 2, 1955.)

\section{2t. H. E. Campbell: On the Casimir operator.}

Let $A$ be either an associative, an alternative or a Lie algebra over an arbitrary field $F$ and let $t\left(R_{x}\right)$ be the trace of the right multiplication $R_{x}$ for $x$ in $A$. It is proved that if the bilinear form $t\left(R_{x} R_{y}\right)$ is nondegenerate then the Casimir operator of the mapping $x \rightarrow R_{x}$ is the identity transformation. If $A$ is associative and $x \rightarrow S_{x}$ is a representation of $A$ and the bilinear form $t\left(S_{x} S_{y}\right)$ is nondegenerate, then $A$ is known to be semi-simple and the representation is completely reducible. If $A$ is also simple and none of the irreducible components of the representation are zero then it is proved that the Casimir operator of $x \rightarrow S_{x}$ is either the identity transformation or zero according as $t\left(R_{x} R_{y}\right)$ is nondegenerate or not. Hence if $A$ is not simple the Casimir operator can be taken to have the form diag $\{I, 0\}$. Similar results are obtained for the case where $A$ is alternative and the characteristic of $F$ does not divide the dimension of $A$. For Lie algebras of prime characteristic the Casimir operator is used together with the enveloping associative algebra of the $R_{x}$ to obtain the Levi theorem for the special case where $A N=0$ ( $N$ the radical of $A$ ), and $A / N$ has nondegenerate Killing form. Research sponsored by U. S. Air Force through the Office of Scientific Research. (Received August 31, 1955.) 


\section{3t. Eckford Cohen: Simultaneous pairs of linear and quadratic} equations in a Galois field.

Let $F$ be a Galois field of order $p^{f}, p$ an odd prime, and let $N_{s}(m, n)$ represent the number of simultaneous solutions in $F$ of the equations $m=\alpha_{1} x_{1}^{2}+\cdots+\alpha_{s} x_{s}^{2}$, $n=\beta_{1} x_{1}+\cdots+\beta_{8} x_{8}$, where $\alpha_{i}, \beta_{i}(i=1, \cdots, s)$ are nonzero elements of $F$. In this paper explicit formulas for $N_{s}(m, n)$ are determined using elementary properties of the generalized Cauchy-Gauss sum. A precise solvability criterion is derived, from which it follows that $s=4$ is the minimum value of $s$ for which $N_{s}(m, n)>0$ for all odd $p$. (Received September 6, 1955.)

\section{Trevor Evans: Some remarks on a paper by R. H. Bruck.}

In a recent paper (Analogues of the ring of rational integers, Proc. Amer. Math. Soc. vol. 6 (1955) pp. 50-58) R. H. Bruck has introduced the concept of right neoring and discussed some properties of these systems. This paper is essentially a commentary on Bruck's paper and some of his results are generalized as follows. The construction of the universal right neoring in Bruck's paper is applied to the free monogenic $V$-loop in any subvariety $V$ of the variety of loops and a complete analogue of Bruck's Theorem 4.1 is obtained for any one of these subvarieties. This construction yields uncountably many right neorings with an identity which generates the additive loop of the right neoring. Conversely, every right neoring with an identity which generates its additive loop can be obtained from a free monogenic $V$-loop by this construction. One possible answer is given to the question raised by Bruck concerning the existence of universal right neorings with free additive loop of arbitrary rank. A brief proof is given of the cancellation properties of the monogenic universal right neoring. Finally, the relationship between right neorings and the logarithmetics of Etherington is discussed. (Received September 6, 1955.)

\section{5t. Bruno Harris: Commuting self-adjoint linear transformations.} Preliminary report.

Let $V$ be a finite dimensional vector space over a field of characteristic not 2 , $B(x, y)$ a hermitian or skew-symmetric nondegenerate scalar product in $V$, and $A$ a self-adjoint linear transformation on $V$. Then: 1 . If $B$ is hermitian, every selfadjoint linear transformation commuting with $A$ is a polynomial in $A$ and 1 if and only if the minimum polynomial of $A$ has degree equal to the dimension of $V$. 2. If $B$ is skew-symmetric, every self-adjoint linear transformation commuting with $A$ is a polynomial in $A$ and 1 if and only if the minimum polynomial of $A$ has degree exactly half the dimension of $V$. These results are analogues of the theorem that a matrix is nonderogatory if and only if every matrix commuting with it is a polynomial in it. The method is to decompose $V$ into a direct sum of mutually orthogonal nonisotropic subspaces which are either cyclic for $A$ (hermitian case) or a sum of two cyclic subspaces (skew-symmetric case). (Received September 6, 1955.)

6t. Bruno Harris: Centralizers in special Jordan algebras. Preliminary report.

Let $J$ be a special Jordan algebra, assumed always of characteristic not $2, K$ a subalgebra. Let $C(K)$ be the set of elements of $J$ commuting with every element of $K$ (in the associative multiplication)-for the algebras considered this is also the set of elements of $J$ that operator-commute with every element of $K$. For $x \in J$, let 
$(x)$ be the subalgebra of $J$ generated by $x$ and the identity element (if it exists). Then if $J$ is special and finite dimensional central simple, $C(C((\chi)))=(\chi)$ for all $\chi \in J$. $C(C((x)))=(x)$ also holds when: $1 . A$ is an involutorial simple associative ring with minimum condition, $J$ is the Jordan algebra (over the subfield of self-adjoint elements of the center) of self-adjoint elements of $A$, and $x$ is nilpotent. $2 . V$ is a vector space of countable dimension over a field, $B(x, y)$ is a nondegenerate hermitian or alternate scalar product in $V, J$ is the Jordan algebra of self-adjoint linear transformations, and $\chi$ is an algebraic linear transformation. The proofs are based on the preceding abstract. (Received September 6, 1955.)

\section{Juris Hartmanis: Two embedding theorems for finite lattices.}

One of the important unsolved problems of lattice theory is the problem of embedding every finite lattice in a finite partition lattice. If a geometry on a set $S$ is defined as a collection of subsets (called lines), such that any two distinct elements of $S$ are contained in one and only one subset and every subset contains at least two distinct elements, then the partition lattice can be considered as the lattice of subspaces of such a geometry. The author shows that every finite lattice can be embedded in the lattice of subspaces of a finite geometry. Using this result it is shown that every finite lattice can be embedded in the lattice of all geometries on a finite set. The lattice of all geometries on a set is shown to be again a lattice of subspaces of a geometry and its structure is similar to that of a partition lattice. This reduces the above mentioned problem to the problem of embedding every finite lattice of geometries in a finite partition lattice. (Received August 29, 1955.)

\section{8t. D. R. Hughes: Partial difference sets.}

Let $I$ be a finite projective plane and $G$ a nontrivial group of collineations of $\Pi$. Let $\Pi_{0}$ be the set of points and lines of $\Pi$ which are fixed by every element of $G$. A point of $\Pi$ which is not in $\Pi_{0}$ but is on a line of $\Pi_{0}$ is called a tangent point; a point which is not a tangent point and is not in $\Pi_{0}$ is called an ordinary point. Dually, tangent line and ordinary line are defined. Suppose there are some ordinary points and lines, and $G$ is transitive and regular on both ordinary points and ordinary lines. Then $\Pi_{0}$ must be one of five types of degenerate sub-planes of $\Pi$, none of which contains more than four points or four lines. Examples of each type exist; for three of the types, II can be either Desarguesian or non-Desarguesian; for one type, the only known finite examples are Desarguesian, and for the other type there is a Desarguesian example of order four, while any larger examples (if they exist) must be non-Desarguesian of order $2^{k}+1$. For each type, the existence of the plane is equivalent to the existence of a "partial difference set" $D$ for the group $G$ : every element of $G$ excepting the elements in certain distinguished subgroups is represented in a unique way as $d_{1} d_{2}^{-1}$ and as $d_{3}^{-1} d_{4}$, where the $d_{i}$ are in $D$. (Received August 29, 1955.)

\section{W. E. Jenner: On the class number of nonmaximal orders in p-adic division algebras.}

Let $D$ be a division algebra of finite dimension over a $p$-adic field and let $O$ be an arbitrary order of $D$ (cf. Compositio Math. vol. 11 (1953) pp. 187-203). Two left (right) ideals $a$ and $b$ of $O$ are said to be equivalent if $a=b \cdot \lambda(a=\lambda \cdot b)$ for some $\lambda \in D$. The number of equivalence classes of left ideals and of right ideals is the same and is finite. This is applied to show that there exist only a finite number of unimodularly inequivalent integral $p$-adic representations of given degree of a finite group, a result 
which follows also from a theorem of Maranda (Canadian Journal of Mathematics vol. 5 (1953) pp. 344-355). (Received September 7, 1955.)

10t. M. D. Marcus and J. M. McGregor: Extremal properties of hermitian matrices.

Let $A$ be a non-negative hermitian (n.n.h) matrix with eigenvalues $\lambda(1) \leqq \cdots$ $\leqq \lambda(n)$. For $r \leqq k \leqq n$ and $x(1), \cdots, x(k)$ a set of orthonormal (o. $\mathrm{n})$ vectors let $\Lambda(r, k)$ and $E(r, k)$ denote the $r$ th elementary symmetric functions (e.s.f) of the $\lambda(i)$ and $(A x(i), x(i)), i=1, \cdots, k$, respectively. Theorem: $\min E(r, k)=\Lambda(r, k)$, $\max E(r, k)=C_{k, r}\left(\sum_{j=1}^{k} \lambda(n-j+1) / k\right)^{r}$ where both $\min$ and $\max$ are taken over all sets of $k$ o.n. vectors $x(1), \cdots, x(k)$. This extreme value result is applied to obtain inequalities for the e.s.f of the eigenvalues of a sum and product of n.n.h matrices. Results on the distribution of the diagonal elements of $A$ with respect to the $\lambda(j)$ as well as a lower bound on the Euclidean norm of a product of arbitrary matrices in terms of the norms and singular values of the factors are also obtained. (Received August 3,1955 .)

\section{Morris Newman: The coefficients of modular forms.}

If $n$ is a non-negative integer, define $p_{r}(n)$ as the coefficient of $x^{n}$ in $\prod_{n=1}^{\infty}\left(1-x^{n}\right)^{r}$; otherwise define $\operatorname{pr}_{r}(n)$ as zero. The following theorem is proved using the elliptic modular functions: Let $r$ be an integer, and $p$ a prime $>3$ such that $r(p+1) \equiv 0$ $(\bmod 24)$. Put $\Delta=r\left(p^{2}-1\right) / 24$. Then the identity $p_{r}(n p+\Delta)=(-p)^{r / 2-1} p_{r}(n / p)$ is valid if and only if $r=2,4,6,8,10,14,26$. This identity furnishes a simple, uniform proof of the three Ramanujan congruences for partitions modulo 5, 7, 11. Identities to prime power moduli of the same type are also obtained. In connection with this work tables of $p_{r}(n)$ were computed on SEAC, the High Speed Electronic Digital Computer of the National Bureau of Standards at Washington, D. C. (Received September 29, 1955.)

\section{M. H. Pearl: On Cayley's parameterization. I.}

Let $A$ be a complex Hermitian (real symmetric) matrix. If $Q$ is a skew-Hermitian (real skew-symmetric) matrix such that $A+Q$ is nonsingular, then $P=(A+Q)^{-1}$ - $(A-Q)$ is a cogredient automorph of $A$ such that $I+P$ and $A$ span the same row spaces (and $d(P)=+1$ ). Conversely, if $P$ is a cogredient automorph of $A$ such that $A$ and $I+P$ span the same row spaces (and if $d(P)=+1$ ), then there exists a skewHermitian (real skew-symmetric) matrix $Q$ such that $A+Q$ is nonsingular and $P=(A+Q)^{-1}(A-Q)$. This result extends the Cayley parameterization to a class of singular matrices. It is also shown that the theorem as stated above does not hold for matrices over a field of characteristic 2. (Received September 12,1955.)

\section{J. J. Price: Some duality theorems. Preliminary report.}

Let $S=(X, F, m)$ be a measure space and $R$ a countable family of matrix-valued functions defined on $S$. Sufficient conditions are found in order that $R$ be essentially a complete system of irreducible representations of a compact group $G$ whose Haar measure is essentially $m$. This result generalizes some known theorems. When the functions in $R$ are one-dimensional, $G$ turns out to be abelian. The result is then equivalent to a theorem of Fine (Pacific Journal of Mathematics vol. 5, pp. 51-59). When $S$ is taken to be a compact group and $R$ a complete system of irreducible representations of $S$, the Tannaka duality theorem is obtained. Several analogous results for finite groups are also found. (Received April 26, 1955.) 


\section{4t. Irving Reiner: Integral Maschke representations.}

Let $A$ be an algebra over the ring $\boldsymbol{D}$ of integers in an algebraic number field $k$. For a prime ideal $\mathfrak{p}$ of $\mathfrak{b}$, let $\bar{k}=\mathfrak{v} / \mathfrak{p}$ be the residue class field, and set $\bar{A}=A / \mathfrak{p} A$. Following Gaschütz (Math. Zeit. vol. 56 (1952) pp. 376-387) and Ikeda (Osaka Math. J. vol. 5 (1953) pp. 53-58), define a Maschke representation of an algebra (over a ring) as a representation which can be split off as direct component from any representation of which it is a constituent. It is shown here that if $\Gamma$ is a representation of $A$ in $\mathbf{v}$, and if for each $\mathfrak{p}$ the induced representation $\bar{\Gamma}$ of $\bar{A}$ in $\bar{k}$ is a Maschke representation, then $\Gamma$ is also a Maschke representation. This reduces the problem of determining whether $\Gamma$ is a Maschke representation over the ring $\mathfrak{D}$, to that of deciding (for those $\mathfrak{p}$ for which $\bar{A}$ is not semi-simple) whether $\bar{\Gamma}$ is a Maschke representation over the field $\bar{k}$. The converse of the above result is also proved, for the case where $A$ is a Frobenius algebra. (Received September 8, 1955.)

\section{ANALYSIS}

15t. F. F. Bonsall: Extreme maximal ideals of a partially ordered vector space.

An extreme maximal ideal of a partially ordered vector space $V$ with an order unit $e$ is one that corresponds to an extreme point of the set of normalized positive linear functionals. The importance of extreme maximal ideals in representation theory has been demonstrated by Kadison [ $A$ representation theory for commutative topological algebra, Memoirs of the American Mathematical Society, no. 7, 1951]. An ideal $J$ of $V$ is said to be perfect if, whenever $x \in J$ and $\epsilon>0$, there exists an element $j_{\epsilon} \in J$ with $-\left(j_{\epsilon}+\epsilon \theta\right) \leqq x \leqq j_{\epsilon}+\epsilon \theta$. It is proved that a maximal ideal is extreme if and only if it is perfect. The set of all perfect ideals behaves, in some ways, like the set of all ideals. In particular, if the only proper perfect ideal of $V$ is $(0)$, then $V$ is one-dimensional. This implies that each proper perfect ideal is contained in a perfect maximal ideal. (Received August 1, 1955.)

16. F. E. Browder: Smoothing processes in the theory of elliptic and hyperbolic differential equations.

If $\mu$ is a bounded measurable function on $E^{n}, k_{\epsilon}(x)=(z \epsilon)^{-n} \prod_{i=1}^{n} \exp \left\{-\left|\epsilon^{-1} x_{i}\right|\right\}$, let $K_{\epsilon}(u)=k * u$. Using various special properties of this smoothing operator, one obtains strengthened and simplified forms of various proofs in the general theory of partial differential equations. A proof is obtained that a weak solution of a strongly elliptic system of equations is a strict solution using only $L^{2}$ energy arguments without assuming that $u$ is $(m-1)$-times strongly-differentiable, where $2 m$ is the order of the system. A simplified form is given of Leray's proof for the existence and properties of solutions of the Cauchy problem for hyperbolic equations. (Received September 8, 1955.)

17. R. B. Davis: Asymptotic solutions of the first boundary value problem for a fourth-order elliptic partial differential equation.

Let $L_{\epsilon}\left(u_{\epsilon}\right) \equiv\left(\epsilon^{2} \Delta+a\right)(\Delta+b) u_{\epsilon}=0$, where $a$ and $b$ are negative constants. Prescribe boundary values for $u_{\epsilon}$ and $\partial u_{\epsilon} / \partial n$. Define $u_{0}$ in the identical fashion, but relaxing the prescribed value for the normal derivative, and setting $\epsilon=0$ in the differential equation. Then $u_{\epsilon}=u_{0}+\tilde{\Psi}+\Omega$, where $\tilde{\Psi}$ is a boundary layer term for which an explicit expression is given, and $\Omega$ and its first derivatives are $O(\epsilon)$ in the sense of a Hilbert space 
norm. The method follows Levinson. Use of the integral norm follows a suggestion of Peter Lax and Paul Berg. (Received June 13, 1955.)

18. T. P. Higgins and W. T. Guy, Jr. (p): On the Hankel transform of higher derivatives.

A formula is developed which expresses the Hankel transform of the $n$th derivative of a function in terms of a finite series of Hankel transforms of the function. (Received September 8, 1955.)

\section{9t. Eugene Leimanis: On a theorem of Poincarê and Malkin.}

The theorem in question is concerned with the existence of periodic solutions of a nonautonomous dynamical system of order $n$, containing a small parameter $\mu$ and being such that the generating system admits an infinity of periodic solutions depending upon $1 \leqq k<n$ parameters $h_{1}, h_{2}, \cdots, h_{k}$ [H. Poincaré, Les méthodes nouvelles de la mécanique céleste, vol. 1, p. 84, Paris, Gauthier-Villars, 1892, and I. G. Malkin, The methods of Lyapunov and Poincare in the theory of nonlinear oscillations, p. 17, Gos. Teh. Teor. Lit., Leningrad-Moscow, 1949 (Russian)]. In this paper an exceptional case, when certain equations $P_{j}\left(h_{1}, h_{2}, \cdots, h_{k}\right)=0, j=1,2, \cdots, k$, (derived from the necessary conditions of periodicity) are satisfied identically with respect to the parameters, is considered and a generalization of the above mentioned basic theorem is obtained. This exceptional case is of importance in some applications to the theory of nonlinear oscillations. (Received August 1, 1955.)

20t. Robert McKelvey: Solution about a regular singular point of a second order linear ordinary differential equation involving a large parameter.

This is an investigation of the differential equation $u^{\prime \prime}-\left[\lambda^{2} \phi^{2}(z)+\lambda z^{-1} \psi(z, \lambda)\right.$ $\left.+z^{-2} \tau\right] u=0,|\lambda|>N$, in a bounded domain $R$ about the origin of $z$, under the assumption that $\phi(z)$ does not vanish in $R$. Both $z$ and $\lambda$ are complex, $\phi(z)$ and $\psi(z, \lambda)$ are analytic, and $\tau$ is constant. $\phi(0)$ is adjusted to 1 . Formal solutions $u=A(z, \lambda) W_{k(\lambda), m}(\xi)$ $+B(z, \lambda) W_{1+k(\lambda), m}(\xi)$ are obtained, where $W(\xi)$ is the Whittaker function, $\xi$ denotes $2 \lambda \int \phi(z) d z$, and $A(z, \lambda), B(z, \lambda)$, and $k(\lambda)$ are formal series $\sum_{j=0}^{\infty} \alpha_{j}(z) / \lambda^{i}, \sum_{j=0}^{\infty} \beta_{j}(z) / \lambda^{i}$, and $\sum_{j=0}^{\infty} k_{j} / \lambda^{i}$. It is shown that, for specific ranges of $\xi$, the expressions obtained from this formal one by replacing $A(z, \lambda), B(z, \lambda), K(\lambda)$ by finite segments are asymptotic approximations to true solutions. (Received August 17, 1955.)

21. W. R. Mann (p), C. L. Bradshaw, and J. G. Cox: Improved approximations to differential equations using low order differences.

The purpose of this paper is to present a method for approximating differential equations with difference equations more closely than is possible with the usual finite difference approaches, but without introducing higher order differences. Though not applicable to all differential equations, it can be used very effectively on some which are of great practical interest. This paper is largely taken up with an illustration of the usefulness of the method in the solution of a two-region, two-dimensional reactor problem which is simple enough to be solved analytically. Numerical data, obtained on the ORACLE of the Oak Ridge National Laboratory, enable a comparison of the analytical solution for the buckling with values obtained by the ordinary difference methods and those obtained by the refined process presented in this paper. The comparison 
shows that the refined process gives considerably greater accuracy than the ordinary difference methods, and the cost in increased labor is comparatively small. (Received August 31, 1955.)

22. Lawrence Markus ( $\mathrm{p}$ ) and R. A. Moore: Disconjugate solutions of linear differential equations with almost periodic coefficients.

One considers the differential equation (L) $y^{\prime \prime}+(-a+b p(x)) y=0$, where $a, b$ are real parameters and $p(x)$ is a real almost periodic function on $-\infty<x<\infty$. Either every solution of $(L)$ is oscillatory or else every (nontrivial) solution is disconjugate (has at most one zero). The set of pairs $(a, b)$ for which $(\mathrm{L})$ is disconjugate is a closed convex set $D$ in the $(a, b)$-plane. Interior to $D$ each equation has a solution basis of the form $y_{i}(x)=\exp \left[(-1)^{i} \alpha x+\int_{0}^{x} \phi_{i}(s) d s\right]$ where the $\phi_{i}(x)$ are almost periodic functions of mean zero. The characteristic exponent $\alpha$ is continuous in the interior of $D$ and when defined by $\lim \sup _{x \rightarrow \infty}(1 / x) \log |y(x)|=\alpha$, it is zero on the boundary of $D$. The domain $D$ is the same for every function $p^{*}(x)$ in the closed hull of $p(x)$. For some such $p^{*}(x)$, the resulting equation $\left(\mathrm{L}^{*}\right)$ on the boundary of $D$ has a positive bounded solution. The domain $D$ varies with the choice of the almost periodic function $p(x)$ in a continuous manner. (Received September 7, 1955.)

23. O. G. Owens: Polynomial solutions of the characteristic value problem for the cylindrical wave equation.

There is a known integral representation for that solution $u(x, y, t)$ (existence assumed) of the cylindrical wave equation, $u_{t t}=u_{x x}=u_{y y}$, which assumes the given boundary values $u\left(x, y,\left(x^{2}+y^{2}\right)^{1 / 2}\right)=\psi(x, y)$ on the characteristic cone $t^{2}=x^{2}+y^{2}$ $(t>0)$. Regarding this formula, the following facts must be established: the partial derivatives exist, are bounded on the characteristic cone, have explicit integral representations, and satisfy the wave equation. A direct deduction from the formula of these facts seems to involve substantial difficulties. However, these complexities are avoidable by means of an indirect analysis based upon the principal theorem of the paper. The theorem asserts that a polynomial solution $u(x, y, t)$ of the characteristic value problem exists whenever the prescribed boundary function $\psi(x, y)$ is a polynomial. (Received September 9, 1955.)

\section{G. O. Peters: Boole polynomials of higher and negative orders.}

Charles Jordan in his book Calculus of finite differences (Budapest, 1939) defines Boole polynomials of order one. The author uses different definitions for the Boole polynomials and Boole numbers, definitions consistent with those for the Bernoulli and Euler polynomials and numbers found in Nörlund's Verlesungen ïber Differenzenrechnung (Berlin, 1924). The author defines the Boole polynomials of degree $\nu$ and order $n$, as the polynomial $\zeta_{\nu}^{(n)}(x)$ satisfying the equation $\nabla^{n} \zeta_{\nu}^{(n)}(x)=x^{(\nu)}$, where $x^{(\nu)}=x(x-1)(x-2) \cdots(x-\nu+1)$ and $\nabla$ is the mean operator, $\nabla=1+\Delta / 2, \nabla f(x)$ $=[f(x+1)+f(x)] / 2$. The Boole polynomial of negative order is defined as the polynomial $\zeta_{\nu}^{(-n)}(x)$, of degree $\nu$, and order $-n$, satisfying the equation $\zeta_{\nu}^{(-n)}(x)=\nabla^{n} x^{(\nu)}$. The Boole numbers are then defined by the equation $\zeta_{\nu}^{(n)}(x)=\sum_{i=0}^{\nu} \nu C_{i}\left(\zeta_{i}^{(n)} / 2^{i}\right) x C^{(\nu-i)}$ $=\left(\zeta^{(n)} / 2+x\right)^{(\nu)}$, where $n$ can be either positive or negative. Some properties of these Appell polynomials are found and the values of the Boole numbers are determined. The symmetry of the polynomials is also found. An interesting polynomial relation is found relating the Euler, Boole, and Bernoulli polynomials of the first kind. (Received October 7, 1955.) 
25. P. P. Saworotnow: Involutions on $H^{*}$-algebra. Preliminary report.

Let $\mathfrak{A}$ be a simple proper $H^{*}$-algebra and let $x \rightarrow x^{x}$ be any involution such that $x^{x}=0$ implies $x=0$ and such that $\left(x^{x}, x^{*}\right) \geqq 0$ for every $x \in \mathscr{A}$ where $x^{*}$ is the adjoint of $x$ (i.e., such that $(x y, z)=\left(y, x^{*} z\right)$ and $\left.(y x, z)=\left(y, z x^{*}\right)\right)$. It is shown that under the above conditions the following is true: (1) There exists a scalar product [,] on $\mathfrak{A}$ defining the original topology such that $[x y, z]=\left[y, x^{x} z\right]$ and $[y x, z]=\left[y, z x^{x}\right]$. (2) There exists a scalar product $\{$,$\} on \mathfrak{A}$ defining the same topology such that $\{x y, z\}$ $=\left\{y, x^{x} z\right\}$ and $\{y x, z\}=\left\{y, z x^{*}\right\}$. (Received August 22, 1955.)

26. C. T. Taam: On the solutions of nonlinear differential equations.

Let $p(t)$ and $q(t)$ be real-valued Lebesgue integrable functions on $[0, a]$ for every $a>0$. The oscillatory property of the solutions of $(\mathrm{A}): u^{\prime \prime}+p u+q u^{3}=0$ is investigated by a comparison method. Various results concerning the zeros and amplitudes of the solutions are obtained. These results are applied to study the periodic solutions of (A). The following result, among others, is established: Let $p$ and $q$ be even periodic functions with a common period $L$ and having positive lower and upper bounds; then, for each positive integer $n$, there exists a periodic oscillatory solution of $(A)$ with period $n L$. A boundary value problem is also solved. Set $p=A+f, q=2(B+g)$. It is shown that if (i) $A$ and $B$ are positive constants, (ii) $f$ and $g$ are of $\mathrm{BV}$ on $[0, \infty)$, (iii) $t f$ and $t g$ are Lebesgue integrable on $[0, \infty)$, then every solution $u$ of (A) is bounded on $[0, \infty)$ and has an asymptotic representation $u=A^{1 / 2} B^{-1 / 2} \phi\left(A^{1 / 2} t+C\right)$ $+e(t)$, where $C$ is a constant, $e(t) \rightarrow 0$ as $t \rightarrow \infty$, and $\phi(t)$ is a periodic function defined by $v^{\prime \prime}+v+2 v^{3}=0, v(0)=0, v^{\prime}(0)=E$ for some $E \neq 0$. This work was supported by the Office of Ordnance Research. (Received September 6, 1955.)

\section{H. F. Weinberger: An upper bound for the free membrane.}

The eigenvalue problem $\Delta u+\mu u=0$ on a region $R$ with $\partial u / \partial n=0$ on the boundary of $R$ is considered. The lowest eigenvalue is always zero, and it is the second eigenvalue $\mu_{2}$ that is of interest. Szegö [Journal of Rational Mechanics and Analysis vol. 3 (1954) p. 343] has shown by means of conformal mapping that if $R$ is two-dimensional and simply connected, its $\mu_{2}$ is at most that of a circle of equal area. An alternate proof of this theorem depending only on a real variables argument is given. It is not necessary to assume that $R$ is simply connected. Furthermore, the proof is easily extended to show that if $R$ is an $N$-dimensional region, its $\mu_{2}$ is at most equal to the $\mu_{2}$ of the $N$-sphere of equal volume. (Received September 9, 1955.)

\section{Applied Mathematics}

28. G. E. Forsythe (p) and Louise W. Straus: The Souriau-Frame characteristic equation algorithm on a digital computer.

$A$ is a regular square matrix of order $n$. Let $F(\lambda)=|A-\lambda I|=(-1)^{n}\left(\lambda^{n}-c_{1} \lambda^{n-1}\right.$ $-\cdots-c_{n}$ ). Let $\lambda_{1}, \lambda_{n}$ be the least, greatest in modulus of the zeros of $F$. Let $P=\left|\lambda_{n}\right| /\left|\lambda_{1}\right|$. In the Souriau-Frame algorithm to get $F(\lambda)$ [see P. Dwyer, Linear computations, Wiley, 1951] one computes $A_{1}=A, c_{1}=$ trace $A, B_{1}=A_{1}-c_{1} I, \cdots, A_{k}$ $=A B_{k-1}, c_{k}=$ trace $A_{k} / k, B_{k}=A_{k}-c_{k} I, \cdots$. Theoretically $c_{k}=0(k \geqq n+1)$. Let a machine word length be $s$. The authors have prepared a single-precision SWAC code $(s=36)$ for the algorithm, using scaled vectors. Tests with several matrices $A$ $(n \leqq 10)$ confirm for $s=36$ the heuristic argument that the round-off error $\Delta c_{k}$ in $c_{k}$ 
behaves roughly thus: $\left(^{*}\right)\left|\Delta c_{k}\right| \doteq 2^{-8-5}\left|\lambda_{n}\right|^{k}$. When $\left(^{*}\right)$ holds, it is shown that $b$ bits of relative accuracy may be expected in $c_{n}$ if and only if $n \log _{2} P \leqq 2 s-2 b+10$, a rather severe restriction. A paper will appear in J. Math. Physics. (Received August 3, 1955.)

\section{Patrick Leehey: The Hilbert problem for an airfoil in unsteady} motion.

Birnbaum's linearization of the equations of motion for unsteady incompressible flow about a thin airfoil in arbitrary accelerated motion leads to a particular Hilbert problem with the complex perturbation velocity $\Phi$ representing a sectionally holomorphic function which vanishes at infinity and satisfies a condition $\Phi^{+}=G \Phi^{-}+g$ on the real axis. The functions $G$ and $g$ have discontinuities at points corresponding to the leading and trailing edges of the airfoil and to the end point of its wake. Following Keldysch and Sedov (cf. Muskhelishvili, Singular integral equations, Moscow, 1946) an expression for $\Phi$ is determined under the condition that $\Phi$ remain bounded in a neighborhood of the trailing edge. This, in turn, yields the well-known singular integral equation of unsteady lifting theory and its inversion. The "continuity" condition of Söhngen (Luftfahrtforschung vol. 17 (1940) p. 402) is shown to be a consequence of the boundedness condition. With the aid of certain results concerning the interchange of integration processes for contours and arcs, Kelvin's circulation theorem is employed to relate the wake vortex distribution to prescribed vertical motions of the airfoil in the form of Wagner's integral equation. (Received September 2, 1955.)

\section{H. D. Mills: Sensitivity of linear programs.}

Let $A=\left\|a_{p q}\right\|$ and $H=\left\|h_{p q}\right\|$ be real $m+1$ by $n+1$ matrices $(p=0,1, \cdots, m$; $q=0,1, \cdots, n)$. Let $V(A)$ be the maximum (value) of the linear function $a_{00}+\sum x_{i} a_{i 0}$ constrained by the $m+n$ linear inequalities $a_{0 i}+\sum x_{i} a_{i j} \geqq 0, x_{i} \geqq 0(i=1, \cdots, m$; $j=1, \cdots, n)$. Let $A^{*}$ be a matrix identical with $A$ except that $a_{00}^{*}=a_{00}-V(A)$. Then if $V(A+\alpha H)$ exists for $0 \leqq \alpha<$ some $\alpha^{0}, \lim _{\alpha \rightarrow 0+}[V(A+\alpha H)-V(A)] / \alpha=$ $V\left(\left\|_{A * 0}^{B} \Lambda^{*}\right\|\right)$. That is, the one-sided directional derivative of the value of the $m+1$ by $n+1$ program $A$ with respect to $H$ is the value of a $2 m+2$ by $2 n+2$ program constructed from $A^{*}$ and $H$ as indicated. (Received September 9, 1955.)

\section{1t. L. E. Payne: On certain multi-valued functions in generalized axially symmetric potential theory.}

To each solution $\phi_{p}$ of the equation of generalized axially symmetric potential theory (1) $\partial^{2} \phi_{p} / \partial x^{2}+\partial^{2} \phi_{p} / \partial y^{2}+(p / y) \partial \phi_{p} / \partial y=0$ ( $p$ any positive real number) there exists a conjugate or stream function $\psi_{p}$ defined up to an additive constant by the relations (2) $y^{p} \partial \phi_{p} / \partial x=\partial \psi_{p} / \partial y, y^{p} \partial \phi_{p} / \partial y=-\partial \psi_{p} / \partial x$. By introducing toroidal coordinates one can represent certain multi-valued conjugate functions in forms which clearly exhibit both their cyclic nature and analytic character. In particular the expressions obtained for the function conjugate to the Neumann's function for the half plane and that for the stream function for a source disk are analytically more transparent than the previously known Bessel function representations (A. Weinstein, Bull. Amer. Math. Soc. vol. 59 (1953)). (Received September 9, 1955.)

32t. Raymond Sedney: Some properties of the three-dimensional boundary layer equations. 
The equations for laminar flow in the boundary layer over a general surface in three dimensions are analyzed in a normal coordinate system. These are not tensor equations, but they do have the invariance properties of subtensors. It is found that the flow is essentially two-dimensional if the external flow streamlines are geodesics on the surface. Physically this is equivalent to a criterion for no "secondary flow." (Received September 12, 1955.)

\section{Domina E. Spencer: Electrodynamic field theory.}

Previous papers (for example, Moon and Spencer, J. Franklin Inst. vol. 257 (1954) pp. 369-382) have presented an electrodynamic equation for the force between two moving charges. This single equation is relativistic in the Galilean sense and may be employed to replace the four Maxwell equations. The present paper shows how the new electrodynamics can be expressed as a field. Equations are developed for scalar and vector potentials, and the new field theory is compared with the electromagnetic theory of Maxwell. (Received September 7, 1955.)

\section{GEOMETRY}

\section{Fred Supnick: Optimal closed paths through certain point sets.}

It is shown in Theorem I that if a finite set of points in $E_{r}$ can be ordered $p_{1}, \cdots, p_{n}$ so that for every set of integers $a, b, c, d$ for which $1 \leqq a<b<c<d \leqq n$ (“ $d()^{\prime}$ " indicates "distance") $d\left(p_{a} p_{b}\right)+d\left(p_{c} p_{d}\right) \leqq d\left(p_{a} p_{c}\right)+d\left(p_{b} p_{d}\right) \leqq d\left(p_{a} p_{d}\right)+d\left(p_{b} p_{c}\right)$, then the shortest and longest (closed, rectilinear) polygons having these and only these points as vertices are $\left(\cdots p_{7} p_{5} p_{3} p_{1} p_{2} p_{4} p_{6} \cdots\right)$ and $\left(\cdots p_{n-5} p_{5} p_{n-3} p_{3} p_{n-1} p_{1}\right.$ - $\left.p_{n} p_{2} p_{n-2} p_{4} p_{n-4} p_{6} \cdots\right)$ ) respectively (edges are the "joins" of adjacent vertices, first and last being considered adjacent). Realizations of the above criterion are constructed. Thus e.g. let $n$ rays $r_{1}, \cdots, r_{n}$ emanating from a point 0 be such that the angles $r_{i} r_{j}\left(<180^{\circ}\right)$ are all equal for $i \neq j$. Let a point $q_{i}$ be selected on $r_{i}(i=1, \cdots, n)$. Let $p_{1}, \cdots, p_{n}$ be that rearrangement of $q_{1}, \cdots, q_{n}$ so that $d\left(0 p_{1}\right) \leqq d\left(0 p_{2}\right) \leqq \cdots$ $\leqq d\left(0 p_{n}\right)$, then $p_{1}, \cdots, p_{n}$ satisfies the conditions of the above theorem. It is shown however that not every finite point set in $E_{r}$ satisfies the conditions of Theorem I. In Theorem II conditions less stringent than those of Theorem I are established, but applicable only for finding the shortest polygon. This permits a wider class of realizations for the minimal case than does Theorem I. The same is done for the maximal case in Theorem III. (Received September 9, 1955.)

\section{TOPOLOGY}

\section{Eldon Dyer: Some examples of quasi-complexes.}

A chainable continuum is a compact (f.o.c.) Hausdorff space having a cofinal family $\Omega$ of finite open coverings such that the elements of each member of $\Omega$ can be linearly ordered in such a way that two of them intersect if and only if they are adjacent in the ordering. The metric chainable continua have been called snake-like by R. H. Bing and include arcs, pseudo-arcs, the closure of the graph of $y=\sin (1 / x), 0<x \leqq 1$, and certain other continua. Any linearly ordered, connected, compact Hausdorff space is also a chainable continuum. In this note it is shown that the cartesian product of the elements of any finite set of chainable continua is a quasi-complex. The term quasi-complex has been defined by S. Lefschetz in Algebraic topology, vol. 27 of the Colloquium Publications of the American Mathematical Society. Lefschetz also shows in the same book that each zero-cyclic quasi-complex has the fixed point 
property. Thus the cartesian product of the elements of any collection of chainable continua has the fixed point property. This generalizes a result announced earlier by the author. (Received September 7, 1955.)

\section{E. E. Floyd: Examples of fixed point sets of periodic maps. II.}

$Y$ will denote a space and $T$ a periodic map on $Y$ of period 6, with fixed point set $F$. An example is given in which $Y$ is a contractible finite complex, and $F$ is not connected. The author also gives an example in which $Y$ is a compact absolute retract, and $F$ is not locally connected. Other examples are given, all showing the eccentricities of fixed point sets of periodic maps of composite period. (Received September 6, 1955.)

37t. L. F. McAauley: $A$ note on naturally ordered sets in semi-metric spaces.

G. T. Whyburn and Zarankiewicz have proved that if $G$ is any naturally ordered collection of disjoint sets in a separable metric space, then at most a countable number of elements $g$ of $G$ can contain a point which is not a condensation point both of the collection $P_{g}$ of all predecessors of $g$ in $G$ and the collection $F_{g}$ of all successors of $g$ in $G$. This note shows that the analogue of such a lemma holds in a hereditarily separable semi-metric topological space $S$; furthermore, a theorem due to G. T. Whyburn [Analytic topology, p. 45 (2.2)] related to this lemma is also true in $S$. Thus, certain theorems which depend upon these fundamental results of Whyburn and Zarankiewicz may be established for spaces which are more general than separable metric spaces. (Received September 9, 1955.)

\section{8t. S. K. B. Stein: Local connectedness and closed continuous maps.}

Let $X$ and $Y$ be a topological space (satisfying the usual 3 axiom on open sets), and $f$ a continuous map from $X$ onto $Y$. Theorem: If $X$ is locally-connected (1.c.) and $f$ is closed, then $Y$ is l.c. The proof consists of showing first that $Y$ is weakly locally connected (w.l.c.) and then employing the classical result that w.l.c. implies 1.c. This enables one to state the Hahn-Mazurkiewicz theorem without any restriction (such as Hausdorff, or metric) on the space $Y$, but instead with the (usually superfluous) restriction that $f$ be closed. Specifically, a necessary and sufficient condition that a space $Y$ be the image of the closed unit interval, $I$, under a closed continuous function is that $Y$ be $t_{1}, t_{4}$, connected, l.c., compact, and have a countable base ( $t_{4}$ does not assume $t_{2}$ ). This theorem suggests defining an equivalence relation $r$ between spaces: $X r Y$ iff these are continuous closed onto maps $f: X \rightarrow Y, g: Y \rightarrow X$. Employing the H.-M. theorem, one shows easily that the spaces equivalent to $I$ are precisely the Peano spaces (i.e. spaces satisfying the above six conditions) containing at least two points. Also, two trianguable spaces are equivalent iff they have the same number of components and the same number of isolated points. For finite spaces the relation $r$ reduces to "homeomorphic." (Received August 8, 1955.)

\section{G. R. Strohl, Jr.: Strongly cyclic Peano spaces.}

A cyclic Peano space $M$ is defined to be strongly cyclic at a point $p$ if, for every infinite sequence of points of $M$ converging to $p$, there is in $M$ a simple closed curve containing infinitely many points of the sequence. $M$ is strongly cyclic if it is strongly cyclic at each of its points. This extends the concept of strongly arcwise connected spaces as studied by Hall and Puckett, Amer. J. Math. vol. 63 (1941) pp. 554-562. 
Results obtained are as follows: (1) a necessary and sufficient condition that $M$ be strongly cyclic is that for every infinite sequence of open sets in $M$, there is in $M$ a simple closed curve intersecting infinitely many of these sets; (2) if $M$ is strongly arcwise connected but not strongly cyclic at a point $p$, there is in $M$ a closed set $D$ and a separation $M-D=\left(\cup K_{i}\right) \cup_{N}$ where the $K_{i}$ form an infinite collection of distinct components of $M-D$ converging to $p$, and $F\left(K_{i}\right)$ consists of exactly two points of $D$. As a corollary, if no two points cut $M$, then $M$ is strongly cyclic. (Received August 9,1955 .)

R. D. SCHAFER, Associate Secretary 